\title{
Negative Autoregulation Controls size Scaling in Confined Gene Expression Reactions
}

Yusuke T. Maeda ( $\square$ ymaeda@phys.kyushu-u.ac.jp)

Kyushu University

\section{Research Article}

Keywords: Gene Expression Reactions, Negative Autoregulation Controls size Scaling, sustain biological systems

Posted Date: November 10th, 2021

DOI: https://doi.org/10.21203/rs.3.rs-1044443/v1

License: (9) This work is licensed under a Creative Commons Attribution 4.0 International License. Read Full License 


\title{
Negative autoregulation controls size scaling in confined gene expression reactions
}

\author{
Yusuke T. Maeda ${ }^{1, *}$ \\ ${ }^{1}$ Department of Physics, Kyushu University, Motooka 744, Fukuoka 819-0395, Japan
}

(Dated: November 3, 2021)

\begin{abstract}
Gene expression via transcription-translation is the most fundamental reaction to sustain biological systems, and complex reactions such as this one occur in a small compartment of living cells. Transcriptional feedback that controls gene expression during mRNA synthesis is a vital mechanism that regulates protein synthesis in cells. There is increasing evidence that the cellular compartment induces steric effects in gene expression reactions. However, the finite-size effect of spatial constraints on feedback regulation is not well understood. Here, we study the confinement effect on transcriptional negative feedback regulation of gene expression reactions using a theoretical model. We find that negative feedback regulation alters the scaling relation of gene expression level on compartment volume, approaching the regular scaling relation without the steric effect. Our findings suggest that negative autoregulatory feedback at the transcription step can dampen the size-dependence of protein expression levels in heterogeneous cell populations.
\end{abstract}

\section{INTRODUCTION}

The micron-sized compartment that separates the cytoplasmic space from the exterior environment is a fundamental feature of living cells $[1,2]$. DNA that stores genetic information is encapsulated in a tiny cellular compartment with a lipid membrane. Catalytic proteins are synthesized by transcription of the genetic information stored in the DNA sequence into messenger RNA (mRNA) and translation of the mRNA sequence into a single chain polymer of amino acids. These complex transcription-translation (TXTL) reactions proceed autonomously under cell-sized confinement of a few microns [3-7]. On the other hand, because protein complexes that regulate TXTL reactions, for example, RNA polymerase or ribosome, have a finite size of a few tens of nanometers, the excluded volume cannot be negligible in a tiny intracellular space [8]. Hence, the question of how the TXTL reactions confined to the small compartment are affected is vital for understanding the physical nature of gene expression under confinement $[9,10]$ and implementing cell-free gene expression in biochemical reactors [11-14].

Past studies have examined gene expression in cellsized water-in-oil emulsions as artificial cells to understand the excluded volume effect in confined TXTL reactions. This approach has shown that in small artificial cells TXTL reactions can be suppressed, and the amount of expressed protein is not proportional to the volume of artificial cells. In contrast, the amount of expressed proteins in large artificial cells increases proportionally to the volume of artificial cells [8]. Such anomalous size-dependence in confined TXTL reactions suggests that the excluded volume effect significantly suppresses protein production under spatial constraints. Indeed, confinement-induced repression needs to be resolved to construct a biochemical factory utilizing cellfree TXTL reactions $[9,10,14]$. What kind of mechanism is required in confined TXTL reactions to sustain the ordinary size dependence even in small artificial cells?

The key to addressing this issue is the regulatory network in the TXTL reactions, in which the amount of expressed proteins is controlled by transcriptional factors [15]. For instance, the autoregulatory feedback where the transcription factor regulates its encoding gene has been identified widely as a "network motif" in gene regulatory networks $[16,17]$. In particular, negative autoregulatory feedback (NAF) control is an abundant network motif. NAF control has broad functions, fast kinetic response [18], suppressing concentration variability [19, 20], mutational robustness [21] and the protein synthesis on demand [22]. However, although there are extensive studies on NAF control in gene regulatory networks in bulk, its regulatory role in confined TXTL reactions is not well understood.

In the present study, we investigate confined TXTL reactions with NAF in a cell-sized compartment by considering a mathematical model. We analyze the size dependence of the amount of protein expressed with the NAF control. The mathematical model shows that the anomalous size-dependent scaling is dampened in the presence of NAF control at the transcriptional level. Such size scaling approaches are close to ordinary volume dependence because mRNA synthesis is suppressed by the excluded volume effect in the small compartment and by the action of NAF control in the large compartment. Our findings may provide insights into the functional role of NAF control in the homeostasis control of the TXTL reaction under the variability of cell size.

\section{RESULTS}

\section{Gene expression reaction in a confined space}

This section presents a mathematical model of a TXTL reaction encapsulated in a cell-sized space. For simplicity, we assume a spherical compartment of radius $R$, in 


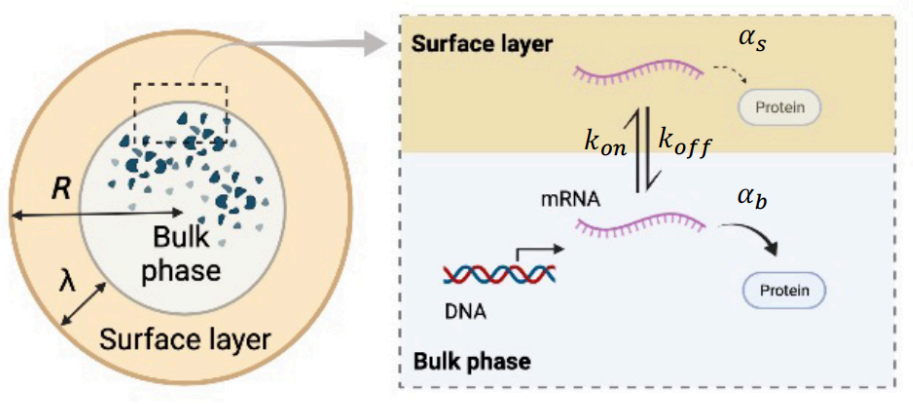

FIG. 1. Schematic illustration of the TXTL reaction. Gene expression is modeled considering the excluded volume effect in a cell-sized spherical compartment. In the bulk phase (blue), the transcription at the reaction rate $\alpha_{r}$ and the translation at the reaction rate $\alpha_{b}$ proceed. On the other hand, in the surface layer (orange) transcription is completely suppressed. Furthermore, mRNA binds to boundary surface from the bulk phase and, in turn, the translation rate drops to $a l p h a_{s}$ in the surface layer. Figure was created with BioRender.

which the molecular system for the TXTL reaction is enclosed (Fig. 1). Among the molecules involved in gene expression, we assume that large protein complexes such as RNA polymerase and ribosomes (typical radius $R_{g}$ ) are subject to steric repulsion against the surface of the compartment.

To consider the excluded volume effect of ribosomes near the boundary, we formulated the transcription reaction from DNA to mRNA and the translation reaction from mRNA to polypeptide protein in the two regions (Fig. 1). First, the surface layer is present beneath the compartment boundary with a thickness of $\lambda$ comparable to the radius $R_{g}$ of the large protein complexes involved in TXTL reactions. Typically, $\lambda$ is on the order of few tens $\mathrm{nm}$, which is sufficiently small compared to radius $R$. Second, these protein complexes capture mRNA inefficiently inside the surface layer; thus, the translation of genetic information from mRNA into protein is likely to be suppressed. In contrast, the active TXTL reaction occurs in a bulk phase free from the excluded volume effect. The mRNAs in each layer then serve as templates for the ribosomal translation process at a different protein production rate, $\alpha_{b}$ for the bulk phase, and $\alpha_{s}$ for the surface layer.

Next, we consider the mRNA concentration in the bulk region $r_{b}(t)$ and the mRNA concentration in the surface layer $r_{s}(t)$ at time $t$. The rate at which mRNA in the bulk region attaches to the surface layer is defined as $k_{o n}$, whereas the rate at which mRNA in the surface layer dissociates into the bulk region is defined as $k_{o f f}$. The mRNA degradation is equal in both the bulk and surface layers, $\gamma_{r}$, and the transcription rate of mRNA is $\alpha_{r}$. Based on the above reactions, the time evolution of
mRNA concentration in the bulk phase is

$$
\frac{d r_{b}}{d t}=\alpha_{r}-k_{o n} r_{b}(t) \frac{S \lambda}{V}+k_{o f f} r_{s}(t)-\gamma_{r} r_{b}(t),
$$

where $S$ and $V$ are the surface area and volume of the confined spherical space, respectively $\left(S=4 \pi R^{2}\right.$ and $V=\frac{4 \pi}{3} R^{3}$ ). By taking the same formulation, the time evolution of mRNA in a surface layer is given by

$$
\frac{d r_{s}}{d t}=k_{o n} r_{b}(t) \frac{S \lambda}{V}-k_{o f f} r_{s}(t)-\gamma_{r} r_{s}(t)
$$

The translation reaction proceeds at a translation rate of $\alpha_{b}$ in the bulk region and at $\alpha_{s}$ in the surface layer, and the surface layer has a lower translation efficiency, that is, $\alpha_{b}>\alpha_{s}$. The average concentration of the protein synthesized in the compartment, $p(t)$, increases with time according to the following equation:

$$
\frac{d p}{d t}=\alpha_{b} r_{b}(t)+\alpha_{s} r_{s}(t)-\gamma_{p} p(t)
$$

where $\gamma_{p}$ is the degradation rate of expressed protein.

The focus of the present study was to reveal the sizedependence of the TXTL reactions at the steady state under confinement, and we then analyzed the steadystate concentrations of mRNA in each region $\left(d r_{b} / d t=0\right.$, $\left.d r_{s} / d t=0\right)$ and that of the expressed protein $(d p / d t=$ $0)$. By solving Eq. (1) and Eq. (2) with setting $d r_{b} / d t=0$ and $d r_{s} / d t=0$, the steady state concentrations of mRNA in bulk phase $\left\langle r_{b}\right\rangle$ and in the surface layer $\left\langle r_{s}\right\rangle$ are

$$
\left\langle r_{b}\right\rangle=\frac{r_{0}}{1+\frac{3 \lambda}{R \tau}}
$$

and

$$
\left\langle r_{s}\right\rangle=\frac{3 \lambda}{R \tau} \frac{r_{0}}{1+\frac{3 \lambda}{R \tau}},
$$

where the mRNA concentration averaged over the compartment is $r_{0}=\alpha_{r} / \gamma_{r}$, and the parameter of mRNA dissociation from the surface layer is $\tau=\left(k_{o f f}+\gamma_{r}\right) / k_{o n}$. We can apply the same calculation to Eq. (3) to obtain the steady-state concentrations of protein summed over two regions $\langle p\rangle$ as follows:

$$
\langle p\rangle=\frac{r_{0}}{\gamma_{p}} \frac{\alpha_{b}+\alpha_{s} \frac{3 \lambda}{R \tau}}{1+\frac{3 \lambda}{R \tau}} .
$$

Eq. (6) indicates the number of the expressed protein $N=\langle p\rangle V$ as

$$
N=\langle p\rangle V=\frac{r_{0}}{\gamma_{p}} \frac{4 \pi R^{3}}{3} \frac{\alpha_{b}+\alpha_{s} \frac{3 \lambda}{R \tau}}{1+\frac{3 \lambda}{R \tau}} .
$$

The dependence of $N$ on the constraint size $R$ is worth noting. The thickness of the surface layer $\lambda$ is a few tens 


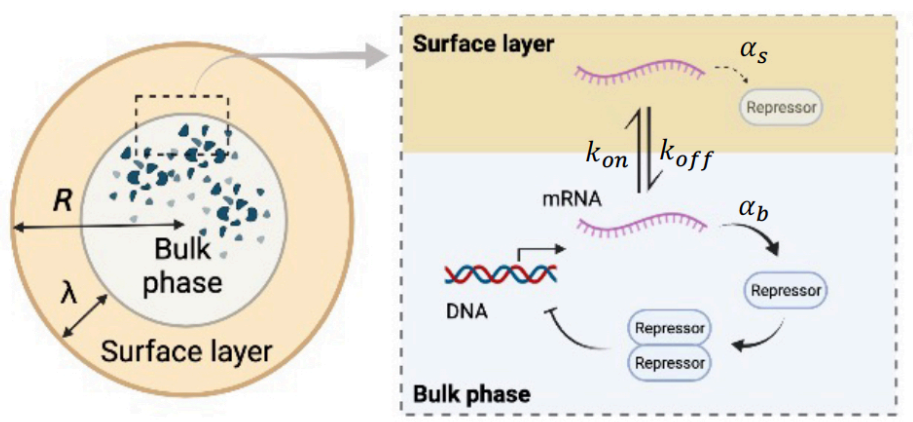

FIG. 2. Schematic illustration of the TXTL reaction with NAF control. The repressor protein synthesized by the TXTL reaction forms a dimer (equilibrium constant $K_{1}$ ). This dimer binds to the operator region of DNA (equilibrium constant $K_{2}$ ). NAF control is realized by reducing the transcription rate of mRNA from the DNA in which the repressor dimer is bound. Figure was created with BioRender.

of nanometers long, and if the size of the confinement is on the micron scale of a cell, $\lambda / R$ can be considered a minute amount. If the translation rate in the surface layer is lower than that in the bulk region $\left(\alpha_{b} \gg \alpha_{s}\right)$, but the mRNA tends to dissociate from the surface layer $\left(k_{o f f}+\gamma_{r}\right) S \lambda \ll V k_{o n}$ (or $\left.\tau \ll 3 \lambda / R\right)$, then Eq. ( 7$)$ is rewritten as

$$
N \approx \frac{\alpha_{b} r_{0}}{\gamma_{p}} \frac{4 \pi R^{3}}{3} \propto R^{3},
$$

meaning that the volume $V$ and the number of protein molecules $N$ follow the same size scaling, $V \propto R^{3}$, and $N \propto R^{3}$. Thus, the excluded volume effect in the surface layer during the translation process is almost negligible.

On the other hand, if the mRNA tends to be trapped in the surface layer $\left(k_{o f f}+\gamma_{r}\right) S \lambda \ll V k_{o n}$ (or $\tau \ll 3 \lambda / R$ ) and its translation is largely suppressed in the surface layer $\left(\alpha_{b} \gg \alpha_{s}\right)$, the number of protein molecules is rewritten as

$$
N \approx \frac{\alpha_{b} r_{0}}{\gamma_{p}} \frac{4 \pi \tau R^{4}}{9 \lambda} \propto R^{4} \lambda^{-1}
$$

Eq. (9) has a dependence on the constraint size $R$ of $N \propto R^{4}$, which is different from the scaling law of Eq. (8) described above. This is because a large number of mRNAs are trapped by a factor of $R / \lambda$ in the translationsuppressing surface layer.

\section{Effect of negative autoregulatory feedback control}

The deviation from the ordinary size scaling shown in the previous section indicates that the TXTL reaction under confinement is affected by surface exclusion volume effects. In this section, we study whether such anomalous size scaling that originates from the encapsulation in cell-sized compartments is affected by transcriptional feedback control in confined TXTL reactions (Fig. 2).
NAF control suppresses the production rate of mRNA at the transcriptional level. The expressed transcription repressor forms a dimeric complex, and the repressor dimer binds to the operator sequence. The complex of repressor dimer-operator DNA inhibits the process of mRNA synthesis, which achieves the repression of mRNA synthesis and, in turn, reduces the expression level of the transcriptional repressor protein (Fig. 2).

The time evolution of the mRNA concentration in the bulk region (Eq. (1)) under NAF control is described by the following equation:

$$
\frac{d r_{b}}{d t}=\frac{\alpha_{r}}{1+K_{1} K_{2} p(t)^{2}}-k_{o n} r_{b}(t) \frac{S \lambda}{V}+k_{o f f} r_{s}(t)-\gamma_{r} r_{b}(t),
$$

where $K_{1}$ is the equilibrium constant for the dimerization of the transcription repressor, and $K_{2}$ is the equilibrium constant for the binding of the repressor dimer to the operator sequence in DNA. As for the transcription in the surface layer, mRNA can be present in the surface layer, but both transcription and translation beneath the boundary hardly occur due to the volume exclusion effect. Hence, the time evolution of the mRNA concentration in the surface layer follows the same equation as Eq. (2).

The steady-state concentration of mRNA in the bulk region is

$$
\left\langle r_{b}\right\rangle=\frac{r_{0}}{\left(1+K_{1} K_{2}\langle p\rangle^{2}\right)\left(1+\frac{3 \lambda}{R \tau}\right)},
$$

and the steady-state concentration of mRNA in the surface layer is

$$
\left\langle r_{s}\right\rangle=\frac{3 \lambda}{R \tau} \frac{r_{0}}{\left(1+K_{1} K_{2}\langle p\rangle^{2}\right)\left(1+\frac{3 \lambda}{R \tau}\right)} .
$$

Furthermore, for protein expression inside the compartment, the transcriptional repressor is degraded at the same rate $\gamma_{p}$ for the bulk region and the surface layer, and the translation from mRNA to protein follows the same equation as Eq. (3). Given that the transcription rate at the steady state can be approximated as $\frac{a l p h a_{r}}{K_{1} K_{2}\langle p\rangle^{2}}$, solving $\langle p\rangle=\alpha_{b}\left\langle r_{p}\right\rangle+\alpha_{s}\left\langle r_{s}\right\rangle$ with Eq. (11) and Eq. (12) yields

$$
\langle p\rangle \approx\left(\frac{r_{0}}{K_{1} K_{2} \gamma_{p}} \frac{\alpha_{b}+\alpha_{s} \frac{3 \lambda}{R \tau}}{1+\frac{3 \lambda}{R \tau}}\right)^{\frac{1}{3}} .
$$

Fig. 3 shows the plot of steady-state protein concentration $\langle p\rangle$ against size $R$ based on Eq. (13). $\langle p\rangle$ drops at a small $R$. Such size-dependent reduction of protein concentration is similar to the TXTL reaction without NAF control (Eq. (6)), but we need to further analyze Eq. (13) to reveal the role of NAF control for the sizedependent TXTL reactions due to the excluded volume effect. 


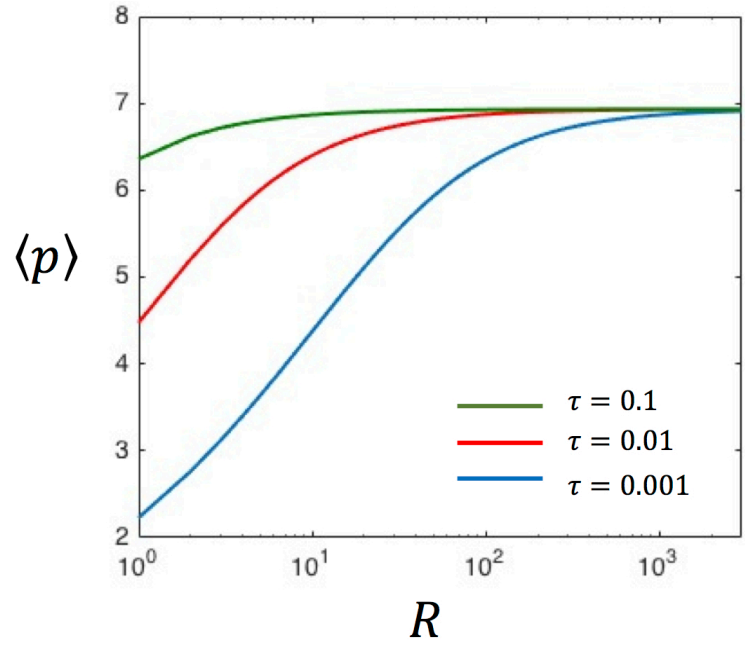

FIG. 3. Steady state protein concentration $\langle p\rangle$ in the TXTL reaction with NAF control. $\langle p\rangle$ is plotted according to Eq. (13) as a function of radius $R$. The three curves represent size dependence of $\langle p\rangle$ for each different parameter $\tau$ (Blue: $\tau=0.001$, Red: $\tau=0.01$, Green: $\tau=0.1$ ). The other parameters are $r_{0}=10, \gamma_{p}=0.03, \alpha_{b}=1, \alpha_{s}=0.001$, and $\lambda=0.01$.

As considered in Eq. (8) in the previous section, the translation rate in the surface layer is lower than in the bulk region $\left(\alpha_{b} \gg \alpha_{s}\right)$. When most of the mRNA is present in the bulk region $\left(k_{o f f}+\gamma_{r}\right) S \lambda \gg V k_{o n}$ (or $\tau \gg 3 \lambda / R$ ), the protein concentration at the steady state is $\langle p\rangle \approx\left(\frac{r_{0}}{K_{1} K_{2} \gamma_{p}} \alpha_{b}\right)^{\frac{1}{3}}$ based on Eq. (13). The number of protein molecules $N=\langle p\rangle V$ in the confined space is

$$
N \approx \frac{4 \pi}{3}\left(\frac{r_{0}}{K_{1} K_{2} \gamma_{p}} \alpha_{b}\right)^{\frac{1}{3}} R^{3} \propto R^{3} .
$$

Similar to the case without NAF control, we find that the volume $V$ and the number of protein molecules $N$ follow the same size scaling $V \propto R^{3}$ and $N \propto R^{3}$. Regular size scaling relation is maintained because the excluded volume effect in the surface layer is almost negligible.

In contrast, when mRNA tends to stay in the surface layer $\left(k_{o f f}+\gamma_{r}\right) S \lambda \ll V k_{o n}$ (or $\tau \ll 3 \lambda / R$ ) and undergo NAF control under the influence of the exclusion volume effect, the protein concentration can be evaluated as $\langle p\rangle \approx\left[\frac{r_{0}}{K_{1} K_{2} \gamma_{p}}\left(\alpha_{s}+\alpha_{b} \frac{R \tau}{3 \lambda}\right)\right]^{\frac{1}{3}}$. Suppose that the TXTL reaction is highly active in the bulk region, whereas the reaction is suppressed in the surface layer $\left(\alpha_{b} \gg \alpha_{s} \frac{3 \lambda}{R \tau}\right)$, the number of expressed proteins $\langle p\rangle V$ is

$$
N \approx \frac{4 \pi}{3}\left[\frac{r_{0}}{K_{1} K_{2} \gamma_{p}}\left(\alpha_{s}+\alpha_{b} \frac{R \tau}{3 \lambda}\right)\right]^{\frac{1}{3}} R^{3} \propto R^{\frac{10}{3}} \lambda^{-\frac{1}{3}} .
$$

The newly obtained size scaling in Eq. (15) differs from the scaling law of Eq. (9) at the case without NAF control. This analysis implies that NAF control alleviates the anomalous volume scaling originating from the ex- cluded volume effect and transforms it into a closely normal size scaling of $R^{10 / 3}$ on the change in compartment size. Without NAF control, a doubling of the volume, such as in cell division, would double the molecular concentration due to gene expression. However, with NAF control, the change in protein concentration at the twofold cell volume was limited to $2^{1 / 3} \approx 1.26$ times. This analysis suggests that the NAF control can limit the possible change in molecular concentration arose from the excluded volume effect to a small variation.

\section{DISCUSSION}

The mathematical model in this study has demonstrated the size-dependent scaling law of gene expression in a microcellular environment and that the scaling exponent can be changed by transcriptional control. In recent years, cell-free extracts have been used to study biological phenomena [1-6]. The exclusion volume effect near the membrane boundary becomes more prominent as the cell size decreases; thus, the confinement effect cannot be ignored. A previous study found that in simple gene expression, the exclusion volume effect near the interface contributes to repression at the translation level, which changes the size-dependent scaling of the compartment at small droplet sizes [8]. The present study extends this to a system with transcriptional autoregulatory feedback, showing that the NAF control of transcriptional regulation is an effective scaling law that avoids the anomalous size-dependent scaling law. In fact, in tiny bacteria such as $E$. coli, network motifs in which negative feedback regulation frequently appear in transcriptional circuits are known $[16,17]$. Thus, the NAF control is an essential regulatory mechanism that contributes to the suppression of size-dependent fluctuations among heterogeneous cell populations. Towards the construction of artificial bioreactors, next important step is to analyze how positive autoregulatory feedback control [23-25], which is another network motif widely identified in transcriptional circuits, controls the TXTL reactions under spatial constraints.

\section{Data availability}

All data generated during the current study are available from the corresponding author on reasonable request.

*ymaeda@phys.kyushu-u.ac.jp

[1] V. Noireaux \& A.P. Liu, The new age of cell-free biology, Ann. Rev. Biomed. Eng. 22, 51-77 (2020). 
[2] A.D. Silverman, A.S. Karim \& M.C. Jewett, Cell-free gene expression: an expanded repertoire of applications, Nat. Rev. Genet. 21, 151-170 (2020).

[3] V. Noireaux \& A. Libchaber, A vesicle bioreactor as a step toward an artificial cell assembly, Proc. Natl Acad. Sci. USA 101, 17669-17674 (2004).

[4] V. Noireaux, Y.T. Maeda \& A. Libchaber, Development of an artificial cell, from self-organization to computation and self-reproduction, Proc. Natl. Acad. Sci. USA 108, 3473-3480 (2011).

[5] S.J. Moore, et al. Rapid acquisition and model-based analysis of cell-free transcription-translation reactions from nonmodel bacteria, Proc. Natl Acad. Sci. USA 115, E4340-E4349 (2018).

[6] K. Hibi, et al. Reconstituted cell-free protein synthesis using in vitro transcribed tRNAs, Commun. Biol. 3, 350 (2020).

[7] E. Cho \& Y. Lu, Compartmentalizing cell-free systems: Toward creating life-like artificial cells and beyond, ACS Synth. Biol. 9, 2881-2901 (2020).

[8] R. Sakamoto, V. Noireaux \& Y.T. Maeda, Anomalous scaling of gene expression in confined cell-free reactions, Sci. Rep. 8, 7364 (2018).

[9] E. Karzbrun, A.M. Tayar, V. Noireaux \& R.H. Bar-Ziv, Programmable on-chip DNA compartments as artificial cells, Science 345, 829-832 (2014).

[10] Z. Izri, D. Garenne, V. Noireaux \& Y.T. Maeda, Gene expression in on-chip membrane-bound artificial cells, ACS Synth. Biol. 8, 1705-1712 (2019).

[11] Y.T. Maeda, T. Nakadai, J. Shin, K. Uryu, V. Noireaux \& A. Libchaber, Assembly of MreB filaments on liposome membranes: A synthetic biology approach, ACS Synth. Biol. 1, 53-59 (2012).

[12] D. Garenne, A. Libchaber \& V. Noireaux, Membrane molecular crowding enhances MreB polymerization to shape synthetic cells from spheres to rods, Proc. Natl Acad. Sci. USA 117 1902-1909 (2020).

[13] R. Marshall \& V. Noireaux, Quantitative modeling of transcription and translation of an all-E. coli cell-free system, Sci. Rep. 9, 11980 (2019).

[14] M. Sobrinos-Sanguino, S. Zorrilla, C.D. Keating, B. Monterroso \& G. Rivas, Encapsulation of a compartmentalized cytoplasm mimic within a lipid membrane by microfluidics, Sci. Rep. 6, 35140 (2016).

[15] M.A. Savageau, Design of molecular control mechanisms and the demand for gene expression, Proc. Natl. Acad. Sci. USA 74, 5647-5651 (1977).

[16] S.S. Shen-Orr, R. Milo, S. Mangan \& U. Alon, Network motifs in the transcriptional regulation network of Escherichia coli, Nat. Genet. 31, 64-68 (2002).

[17] U. Alon, Network motifs: theory and experimental approaches, Nat. Rev. Genet. 8, 450-461 (2007).

[18] N. Rosenfeld, M.B. Elowitz \& U. Alon, Negative autoregulation speeds the response times of transcription net- works, J. Mol. Biol. 323, 785-793 (2002).

[19] A. Becskei \& L. Serrano, Engineering stability in gene networks by autoregulation, Nature 405, 590-593 (2000).

[20] D.W. Austin, M.S. Allen, J.M. McCollum, R.D. Dar, J.R. Wilgus, G.S. Sayler, N.F. Samatova, C.D. Cox \& M.L. Simpson, Gene network shaping of inherent noise spectra, Nature 439, 608- 611 (2006).

[21] C.M. Denby, J.H. Im, R.C. Yu, C.G. Pesce \& R.B. Brem, Negative feedback confers mutational robustness in yeast transcription factor regulation, Proc. Natl. Acad. Sci. USA 109, 3874-3878 (2012).

[22] E. Franco, G. Giordano, P-O. Forsberg \& R.M. Murray, Negative autoregulation matches production and demand in synthetic transcriptional networks, ACS Synth. Biol. 3, 589-599 (2014).

[23] A. Becskei, B. Seraphin \& L. Serrano, Positive feedback in eukaryotic gene networks: cell differentiation by graded to binary response conversion, EMBO J. 20, 2528-2535 (2001).

[24] F.J. Isaacs, J. Hasty, C.R. Cantor \& J.J. Collins, Prediction and measurement of an autoregulatory genetic module, Proc. Natl. Acad. Sci. USA 100, 7714-7719 (2003).

[25] Y.T. Maeda \& M. Sano, Regulatory dynamics of synthetic gene networks with positive feedback, J. Mol. Biol. 359, 1107-1124 (2006).

\section{Acknowledgements}

The author thanks K. Kaya for conducting the preliminary experiment that was the basis of this study. Graphic illustration was created with BioRender software. This work was supported by Grant-in-Aid for Scientific Research on Innovative Areas "Molecular Engines" JP18H05427, Grant-in-Aid for Scientific Research (B) JP20H01872, Grant-in-Aid for Challenging Research JP21K18605 from MEXT.

\section{Author contributions statement}

Y.T.M. designed research, conceived theoretical modeling and wrote the manuscript.

\section{Additional information}

Competing financial interests. The authors declare no competing interests. 